\title{
Sample container for high-resolution neutron imaging of spent nuclear fuel cladding sections
}

\author{
Cite as: Rev. Sci. Instrum. 91, 056103 (2020); doi: 10.1063/1.5143226 \\ Submitted: 20 December 2019 • Accepted: 9 April 2020 • \\ Published Online: 11 May 2020
}
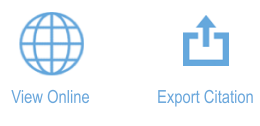

and Liliana I. Duarte ${ }^{2, a}$

\begin{abstract}
Pavel Trtik, ${ }^{1, \text { a) }}$ Robert Zubler, ${ }^{2}$ Weijia Gong, ${ }^{2, b)}$ Robin Grabherr, ${ }^{3}$ Johannes Bertsch, ${ }^{2}$
AFFILIATIONS

${ }^{1}$ Laboratory for Neutron Scattering and Imaging (LNS), Paul Scherrer Institut, CH-5232 Villigen PSI, Switzerland

${ }^{2}$ Laboratory for Nuclear Materials (LNM), Paul Scherrer Institut, CH-5232 Villigen PSI, Switzerland

${ }^{3}$ Abteilung Hotlabor (AHL), Paul Scherrer Institut, CH-5232 Villigen PSI, Switzerland
\end{abstract}

\begin{abstract}
a) Authors to whom correspondence should be addressed: pavel.trtik@psi.ch and liliana.duarte@psi.ch
${ }^{b}$ Now at: Northwestern Polytechnical University, Xi'an, People's Republic of China.
\end{abstract}

\begin{abstract}
In this work, for the first time, high-resolution neutron imaging (true spatial resolution of $13 \mu \mathrm{m}$ ) is used for irradiated nuclear fuel cladding, applying an adapted procedure for transfer, handling, and measurements of highly radioactive samples in combination with the neutron microscope detector at Paul Scherrer Institut. A sample container referred to as an active box for high-resolution neutron imaging of highly active spent nuclear fuel cladding sections was developed. Sections of unirradiated and irradiated cladding of duplex type, having a liner, with hydrogen average concentrations of $420 \mathrm{wppm}$ and $450 \mathrm{wppm}$ were investigated using this device. The irradiated cladding originated from a fuel rod operated for five cycles in a Swiss pressurized water reactor. The irradiated cladding sample was measured inside the active box. Long circumferential hydride accumulations were revealed together with notable hydride precipitation at the liner-substrate interface. Measurements of the unirradiated cladding in air and inside the active box delivered consistent results, confirming the applicability of the developed device for high-resolution neutron imaging.
\end{abstract}

Published under license by AIP Publishing. https://doi.org/10.1063/1.5143226

Neutron imaging is a powerful tool for the assessment of hydrogen distribution and concentration in zirconium based materials. $^{1-3}$ The hydrogen distribution in zirconium based materials is usually investigated using unirradiated samples, and reports on neutron radiography of radioactive, irradiated samples are scarce. ${ }^{4,5}$ Recently, the spatial resolution of neutron imaging was improved down to the sub-5 micrometer domain in $2 \mathrm{D}^{6}$ and about $10 \mu \mathrm{m}$ in $3 \mathrm{D}^{7}$ in the framework of a Neutron Microscope (NM) project. $^{8-10}$ Because such resolution is compatible with the size of common hydride packets, the advanced highresolution neutron imaging detector provides an excellent nondestructive tool for the quantification of hydrogen in nuclear fuel claddings.

Hydrogen concentrations in the zirconium-based claddings can often be non-uniform because of the high mobility of hydrogen interstitial atoms. When the hydrogen concentration in zirconium alloys reaches the solubility limit, the hydrogen precipitates into platelet-like zirconium-hydrides. Hydrides are brittle, and their accumulation or unfavorable orientation can pose a risk to the nuclear fuel rod integrity. In recent works, the assessment of hydrogen migration under stress and different cooling rates in duplex Zircaloy cladding tubes with an external liner was investigated. ${ }^{11}$

It can be shown that the liner in these claddings acts as a sink for hydride formation. In addition, high-resolution neutron radiography was used to quantify the hydrogen diffusion under stress in Zircaloy-4. ${ }^{12}$

However, none of the samples investigated in the publications $^{6-12}$ have been irradiated and were thus not radioactive. Consequently, the mentioned results reflect neither the effects of irradiation-induced damage nor the influence of the heat flux through a fuel cladding being operated in a nuclear reactor. Clearly, the evaluation of hydrogen and hydride concentrations is of very high importance in irradiated claddings, too. Consequently, there is a considerable interest of utilities, regulators, and fuel manufacturers in the assessment of hydrogen/hydride distribution in nuclear fuel claddings at various stages of their life cycle. However, the high 
radioactivity of used nuclear fuel cladding tubes brings several challenges for the experimental procedure of high-resolution neutron imaging-possibly the only tool to perform such a task through remote sensing.

For a safe transfer of the radioactive sample to the neutron beamline, for the handling and positioning of the sample in the experimental hutch, an appropriate sample enclosure, i.e., a specifically designed container, is required to avoid any contamination and radiation safety issues. In addition, the sample container is required to have the least influence on the quality of the high-resolution neutron images. The sample container has to allow easy loading and positioning of irradiated samples into the container, being performed in the PSI Hotlab facility, ${ }^{13}$ and applying respective safety precautions such as shielding, wearing protection mask, and using long tweezers. Furthermore, the sample container should be easily transferrable from the Hotlab to PSI's SINQ ${ }^{14}$ experimental hall in a suitable shielding cask and later into the beamline hutch. There, the sample container needs to be unloaded from the cask and positioned onto the sample stage of the NM; this again has to be possible with long tweezers. In addition, for performing high-resolution neutron imaging, the Zircaloy cladding sample has to be fixed inside the container stably and must allow for an easy alignment of the tube axis parallel to the neutron beam. Due to the neutron beam divergence, the container should allow for positioning the sample as close as possible to the scintillator screen of the neutron imaging detector to avoid an increase in geometrical blur and thus deterioration of the spatial resolution. The attenuation of the neutron beam by the container should be minimized.

The active box fulfilling all these requirements was designed and produced in-house at PSI. Figures $1(\mathrm{a})-1$ (c) show the disassembled active box, consisting of three pieces and an O-ring. It can accommodate different types of cladding tubes with diameters up to $12 \mathrm{~mm}$. Aluminum was the material of choice due to its high neutron transparency. While the structure of the active box is relatively massive to assure mechanical stability, the front and backside consist of rather thin aluminum sheet material $(0.5 \mathrm{~mm})$ to minimize neutron attenuation and to allow sample positioning as close as possible to the imaging detector.

The cladding material used in this pilot study was a commercial duplex cladding produced by Framatome, denoted as DX-D4, consisting of a Zircaloy-4 substrate with an outer liner. Unirradiated and irradiated samples are denoted as $D X-D 4 \_u$ and $D X$ $D 4 \_i$, respectively. The inner and outer diameters of the samples were $9.25 \mathrm{~mm}$ and $10.75 \mathrm{~mm}$, respectively. The chemical composition of the DX-D4 substrate is $\mathrm{Zr}-1.5 \% \mathrm{Sn}-0.2 \% \mathrm{Fe}-0.1 \% \mathrm{Cr}$, and the liner composition differs from the substrate by a reduced tin and increased iron and chromium content. The unirradiated $D X-D 4 \_u$ was charged with 420 wppm of hydrogen using a Sieverts-type apparatus at $440^{\circ} \mathrm{C}$. After hydrogenation, a homogenization treatment was performed, subsequently a heat treatment at $400{ }^{\circ} \mathrm{C}$ for $10 \mathrm{~h}$ with a cooling rate of $0.5^{\circ} \mathrm{C} / \mathrm{min}$ was applied.

The hydrogen measurements of the samples were performed by hot vacuum extraction (HVE) with a shielded hydrogen determinator (LECO, Inc., model RH402). The irradiated sample was cut from a fuel rod irradiated in the Swiss pressurized water reactor of Gösgen, KKG. The DX-D4 cladding has been subjected to five reactor cycles; the rod reached an average burn-up of about
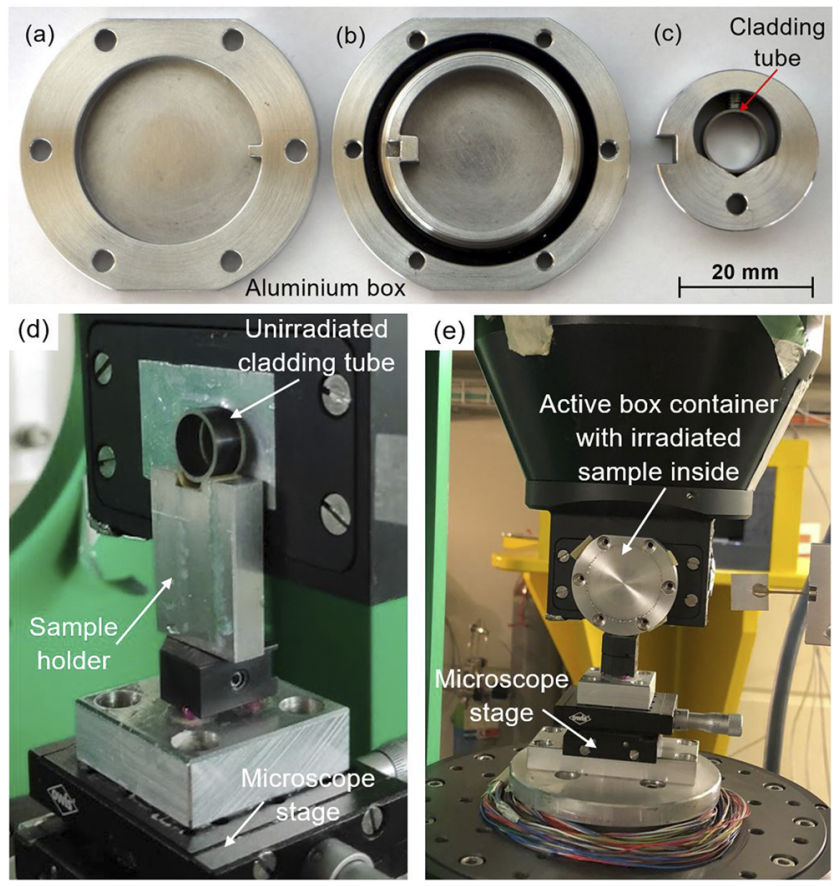

FIG. 1. Aluminum active box with a diameter of $45 \mathrm{~mm}$ : (a) backside, (b) front side, and (c) inside sample holder. Photograph of the NM stage with the sample holder for the unirradiated sample (d) measured in air and with the (e) active box container for irradiated samples.

$70 \mathrm{MWd} / \mathrm{kgU}$. Before testing, the cladding section was cleaned by mechanical and chemical removal of the remaining $\mathrm{UO}_{2}$ fuel. The HVE measurement of a sample that was cut at a rod elevation close to the test specimen $\left(D X-D 4 \_i\right)$ revealed an overall concentration of 446 wppm of hydrogen. For the active box containing the irradiated sample, the dose rates measured in contact and at $10 \mathrm{~cm}$ distance were $10 \mathrm{mSv} / \mathrm{h}$ and $0.83 \mathrm{mSv} / \mathrm{h}$, respectively. The pilot experiments were divided into two parts. First, the performance of the active box was tested using the unirradiated sample $D X-D 4 \_u$. Subsequently, the active box with the irradiated sample $D X-D 4 \_i$ was measured. In both cases, the NM was utilized at the thermal neutron instrument POLDI, ${ }^{15}$ as shown in Figs. 1(d) and 1(e). The chopper of the instrument was levitating in the parking position fully opening the beam. The upstream vertical slits of the instrument collimation system were opened to $18 \mathrm{~mm}$. The downstream horizontal and vertical slits were both opened to $2.5 \mathrm{~mm}$. The slits were placed at $545 \mathrm{~mm}$ distance from the detector, thus providing a collimation ratio of $\mathrm{L} / \mathrm{D}$ $=218$. The neutron microscope detector has been equipped with a $3.5 \mu \mathrm{m}$ thick scintillator based on the isotopically enriched 157gadolinium oxysulfide screen ${ }^{8}$ with an enhanced light output. ${ }^{9}$ The resulting effective pixel size of all the acquired images was equal to $2.7 \mu \mathrm{m}$. Figure 1 (d) shows a detailed view of the unirradiated sample in air and Fig. 1(e) the active box, both positioned on the sample stage in front of the scintillator screen of the NM detector.

The unirradiated sample $D X-D 4 \_u$ was imaged both in air [Fig. 2(a)] and inside the active box [Fig. 2(b)] to compare the resulting images, thus evaluating the degradation in resolution due to the 


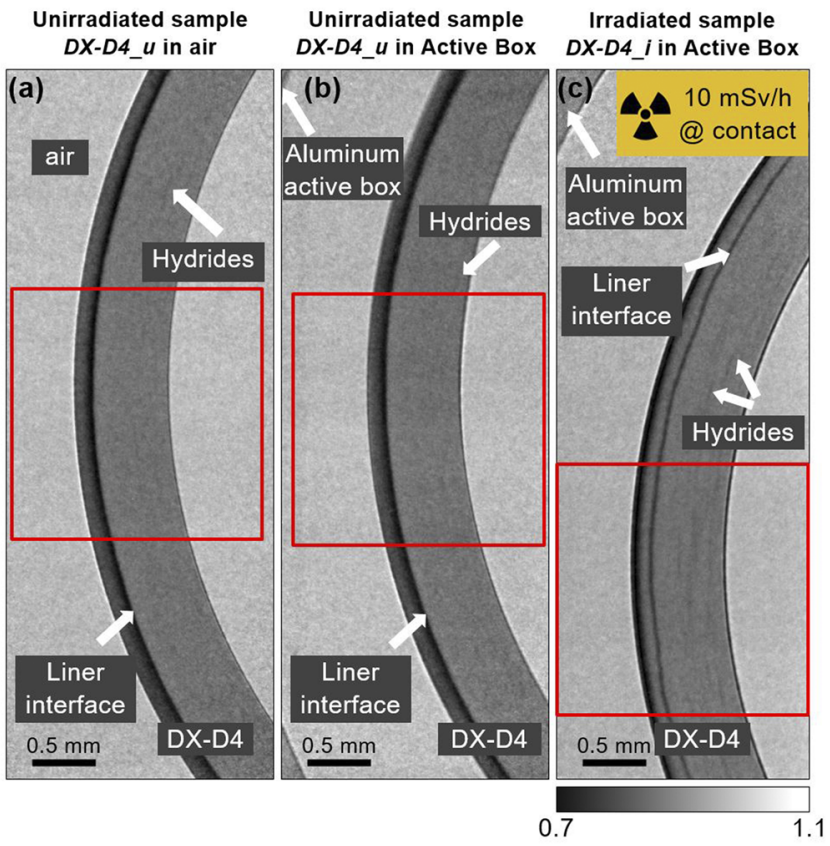

FIG. 2. High-resolution neutron radiographs of segments of nuclear fuel cladding tubes. Inactive sample imaged: (a) in air as a reference image and (b) enclosed in the active box. (c) Irradiated sample exhibiting about $10 \mathrm{mSv} / \mathrm{h}$ dose rate at contact distance enclosed in the active box.

active box. This is an important step for eventually assessing the results of the irradiated sample, as this specimen cannot be measured in air. Figure 2(c) shows the neutron radiography of the irradiated sample $D X-D 4 \_i$, enclosed in the active box. In the images with the active box, i.e., Figs. 2(b) and 2(c), a thick aluminum part of the active box is observed at the left upper corners. The thin central part of the active box attenuates the neutron beam by only $~ 1 \%$ and thus has a negligible influence on the contrast-to-noise ratio of the resulting images of the sample. This is important, in particular, taking into account the fact that the exact same sample container cannot be unloaded and re-measured for reference within a reasonable time. The spatial resolution with and without active box was evaluated by Fourier ring correlation $(\mathrm{FRC})$ of the center part $(708 \times 708$ pixel in size) of the images shown in Fig. 2. While the $D X-D 4 \_u$ sample image in air exhibits the spatial resolution of $12.8 \mu \mathrm{m}$, resolution of the image using the active box is equal to $13.4 \mu \mathrm{m}$. The FRC spatial resolution of the irradiated sample $D X-D 4 \_i$ was assessed to be 13.1 $\mu \mathrm{m}$. It should be noted here that while the derived FRC spatial resolution values are slightly higher than those reported previously, the important issue is that they are very close to each other, thus proving that the designed active box has a very limited negative influence on the spatial resolution. The slightly lower values of the resolution for the cases of samples in the active box are likely caused by the $\sim 0.5 \mathrm{~mm}$ larger sample-to-scintillator screen distance. The lower absolute values of the resolution for all samples are likely due to the fact that the previous assessment was performed on images of the high-contrast gadolinium test object.

Higher attenuation (dark) areas in the neutron images are predominantly related to the increased hydrogen concentration and accumulation of hydrides, as shown in Fig. 2. The related hydride distribution and accumulation effects have been reported in Refs. 11 and 12. In the presented neutron images, hydrides appear as thin darker lines in the substrate, and higher hydride concentrations are located at the liner-substrate interface.

In conclusion, the sample container referred to as an active box for highly activated zirconium based nuclear cladding tube specimens was developed for the purpose of the high-resolution neutron imaging using the neutron microscope with a high-resolution detector at PSI. The combination of radioactive sample analysis and high-resolution neutron imaging is novel. It is demonstrated that the sample container fulfills all the requirements necessary for the highresolution neutron imaging of irradiated samples. The active box has been tested in a pilot study using both unirradiated and irradiated Zircaloy type tube samples. The neutron radiography images with and without active box are of comparable quality.

The authors would like to thank Kernkraftwerk Gösgen, KKG, for the possibility to use the irradiated sample material for testing. Framatome provided the unirradiated material. The project was co-financed by the Swiss Expert Group Fuels and swissnuclear. In addition, the technical support of the radiation protection officers and the support from PSI Hotlab are gratefully acknowledged. The authors kindly thank Professor Markus Strobl (PSI, Switzerland) for proofreading the manuscript. This work is based on experiments performed at the Swiss Spallation Neutron Source (SINQ) at the Paul Scherrer Institute, Villigen, Switzerland.

\section{REFERENCES}

${ }^{1}$ M. Grosse, J. ASTM Int. 8, 103251 (2011).

${ }^{2}$ M. Grosse, M. van den Berg, C. Goulet, E. Lehmann, and B. Schillinger, Nucl. Instrum. Methods Phys. Res., Sect. A 651, 253-257 (2011).

${ }^{3}$ N. L. Buitrago, J. R. Santisteban, A. Tartaglione, J. Marín, L. Barrow, M. R. Daymond, M. Schulz, M. Grosse, A. Tremsin, E. Lehmann, A. Kaestner, J. Kelleher, and S. Kabra, J. Nucl. Mater. 503, 98-109 (2018).

${ }^{4}$ A. E. Craft, D. M. Wachs, M. A. Okuniewski, D. L. Chichester, W. J. Williams, G. C. Papaioannou, and A. T. Smolinski, Phys. Procedia 69, 483-490 (2015).

${ }^{5}$ P. Vontobel, M. Tamaki, N. Mori, T. Ashida, L. Zanini, E. H. Lehmann, and M. Jaggi, J. Nucl. Mater. 356, 162-167 (2006).

${ }^{6}$ P. Trtik and E. H. Lehmann, J. Phys.: Conf. Ser. 746, 012004 (2016).

${ }^{7}$ P. Trtik, MethodsX 4, 492-497 (2017).

${ }^{8}$ P. Trtik and E. H. Lehmann, Nucl. Instrum. Methods Phys. Res., Sect. A 788, 67-70 (2015).

${ }^{9}$ J. Crha, J. Vila-Comamala, E. Lehmann, C. David, and P. Trtik, MethodsX 6, 107-114 (2019).

${ }^{10}$ P. Trtik, M. Meyer, T. Wehmann, A. Tengattini, D. Atkins, E. H. Lehmann, and M. Strobl, Mater. Research Forum 15, 23-28 (2020).

${ }^{11}$ W. Gong, P. Trtik, A. W. Colldeweih, L. I. Duarte, M. Grosse, E. Lehmann, and J. Bertsch, J. Nucl. Mater. 526, 151757 (2019).

${ }^{12}$ W. Gong, P. Trtik, S. Valance, and J. Bertsch, J. Nucl. Mater. 508, 459-464 (2018).

${ }^{13}$ R. Zubler, P. Trtik, J. Bertsch, and L. I. Duarte, extended abstract presented at 56th Annual Meeting on Hot Laboratories and Remote Handling, Mamallapuram, Tamil Nadu, India, September 8-12, 2019.

${ }^{14}$ B. Blau, K. N. Clausen, S. Gvasaliya, M. Janoschek, S. Janssen, L. Keller, B. Roessli, J. Schefer, P. Tregenna-Piggott, W. Wagner, and O. Zaharko, Neutron News 20(3), 5-8 (2009).

${ }^{15}$ U. Stuhr, Nucl. Instrum. Methods Phys. Res., Sect. A 545, 319-329 (2005).

${ }^{16}$ C. Grünzweig, G. Frei, E. Lehmann, G. Kühne, and C. David, Rev. Sci. Instrum. 78, 053708 (2007). 